\title{
IV. Entrenamiento muscular de las extremidades inferiores en el paciente con enfermedad pulmonar obstructiva crónica
}

\author{
DIEGO VARGAS B.*, OSVALDO CABRERA R.*, FRANCISCO ARANCIBIA H.**,***, \\ MARÍA ANGÉLICA ELORRIETA G.*, CRISTIÁN OLAVE C.* y ROBERTO TORRES T.*
}

Lower extremity exercise training in the rehabilitation of patients with chronic obstructive pulmonary disease

Several studies have shown that poor exercise tolerance in Chronic Obstructive Pulmonary Disease (COPD) patients is multifactorial in origin. However, a major exercise-limiting factor in COPD is peripheral muscle dysfunction, particularly the muscles of the lower extremities, characterized by atrophic muscles and reduced fatigue resistance due to morphological and metabolic alterations of peripheral muscles. This chapter therefore evaluated the scientific evidence regarding the beneficial effect of lower extremities exercise in the pulmonary rehabilitation in COPD patients. The technical characteristics of this exercise training were also reviewed. Exercise training of lower limbs was recommended in respiratory rehabilitation of COPD patients. The lower extremities muscle exercise training provides significant benefits to patients with COPD in terms of reduction of dyspnea and improvemet in exercise capacity and in quality of life (quality level of evidence $=A$, strong recommendation). Higher-intensity exercise training and with interval exercise of the lower extrmities produces greater physiological benefits.

Key words: Lower extremities exercise, respiratory rehabilitation, peripheral muscle dysfunction, exercise training, COPD.

\section{Resumen}

Diversos estudios han demostrado que la pobre tolerancia al ejercicio de los pacientes con Enfermedad Pulmonar Obstructiva Crónica (EPOC) es de origen multifactorial. Sin embargo, un importante factor limitante del ejercicio en los pacientes con EPOC es la disfunción muscular periférica, sobre todo de los músculos de las extremidades inferiores, que se caracteriza por atrofia muscular y reducida resistencia a la fatiga dado por alteraciones morfológicas y metabólicas de los músculos periféricos. En este capitulo se evaluó la evidencia científica que existe en cuanto a los beneficios del entrenamiento muscular de extremidades inferiores (EEII) en la rehabilitación respiratoria en pacientes con EPOC. También se revisan las características técnicas de dicho entrenamiento. Se recomienda la realización de entrenamiento muscular de EEII en rehabilitación respiratoria de pacientes con EPOC. El entrenamiento muscular de extremidades inferiores otorga significativos beneficios a los pacientes con EPOC en cuanto a disminuir la disnea, mejorar la capacidad de ejercicio y la calidad de vida (calidad de la evidencia A, recomendación fuerte). El entrenamiento de EEII de alta intensidad y con intervalos produce significativos beneficios fisiológicos.

Palabras clave: Ejercicio de extremidades inferiores, rehabilitación respiratoria, disfunción muscular periférica, entrenamiento muscular, EPOC.

\footnotetext{
* Unidad de Kinesiología, Instituto Nacional del Tórax.

** Servicio de Medicina Respiratoria y Unidad de Paciente Crítico, Instituto Nacional del Tórax.

***Unidad de Respiratorio, Clínica Santa María.
} 


\section{Introducción}

En la enfermedad pulmonar obstructiva crónica (EPOC) moderada y severa la disnea es el síntoma más común, lo cual ocasiona disminución progresiva de la capacidad de ejercicio del individuo hasta limitarlo en las actividades más simples de la vida cotidiana, ya que el ejercicio puede empeorar la disnea ${ }^{1,2}$. Esto conduce a una pérdida de la autonomía del paciente desarrollándose un severo grado de invalidez. En esta situación desfavorable, el progresivo desacondicionamiento físico asociado a la inactividad determinan que el paciente con EPOC entre en un círculo vicioso en el cual cuanto más disnea presenta el paciente es menor su movilidad y viceversa. La única posibilidad de romper este círculo vicioso es realizando ejercicio físico de forma controlada y sistematizada ${ }^{3}$.

Diversos estudios han demostrado que la pobre tolerancia al ejercicio de los pacientes con EPOC es de origen multifactorial e incluye los siguientes aspectos: 1) Limitación ventilatoria; 2) Disfunción de la musculatura periférica; 3) Daño cardiovascular (limitación cardiovascular); 4) Trastornos nutricionales; y 5) Factores psicológicos ${ }^{4-8}$.

Sin embargo, existen evidencias que la fatiga muscular periférica, en particular de los músculos de las extremidades inferiores (EEII), tiene un papel muy importante en la limitación al esfuerzo físico de los pacientes con EPOC. En 1992, Killian y cols 9 , publicaron un documento histórico en el cual demostraron que los pacientes con EPOC, independientemente de la intensidad de la obstrucción bronquial, presentaban en forma frecuente y durante la realización de un esfuerzo físico (protocolo estandarizado en bicicleta), dolor y fatigabilidad de las piernas además de disnea. Este informe fue la base de los fundamentos científicos para iniciar e investigar la disfunción muscular de las extremidades inferiores en los pacientes con enfermedad pulmonar obstructiva crónica. Posteriormente, Güell y cols ${ }^{10}$, demostraron que la fatiga muscular de extremidades inferiores aparecía en el 53\% de los pacientes con EPOC, a pesar de que sólo el 33\% refería dolor en las piernas durante la actividad física.

Los pacientes con enfermedad pulmonar obstructiva crónica tienen un grado más o menos importante de atrofia muscular, lo cual se debe a varios factores, como son: la inmovilidad, la desnutrición, la hipoxemia, y el uso de corticoides entre otros factores. Se ha demostrado que la atrofia muscular provoca disminución del número de mitocondrias, reducción de la actividad enzimática del músculo esquelético y una serie de alteraciones en la microcirculación de la fibra muscular. Todo ello, dificulta el intercambio gaseoso de la fibra muscular y provoca una disminución de la capacidad metabólica, favoreciendo la fatiga muscular durante el ejercicio físico ${ }^{11,12}$.

\section{Preguntas}

1. ¿Está indicado el entrenamiento muscular de extremidades inferiores en pacientes con enfermedad pulmonar obstructiva crónica?

2. ¿El entrenamiento muscular de extremidades inferiores disminuye la disnea y aumenta la capacidad de realizar actividad física en pacientes con EPOC?

3. ¿Qué técnicas de entrenamiento muscular de extremidades inferiores han demostrado ser efectivas?

\section{Pacientes}

Pacientes con enfermedad pulmonar obstructiva crónica estables que presentan disnea y limitación de la capacidad de realizar ejercicio a pesar del tratamiento farmacológico óptimo.

\section{Intervención}

Entrenamiento muscular de extremidades inferiores (EM-EEII) en pacientes con EPOC.

\section{Objetivo}

Evaluar si el entrenamiento muscular de extremidades inferiores alivia la disnea, aumenta la capacidad de realizar ejercicio y mejora la calidad de vida en los pacientes con EPOC. Además, evaluar las mejores técnicas de entrenamiento muscular periférico.

\section{Resumen de la evidencia}

El entrenamiento muscular de las extremidades inferiores ha sido el mejor estudiado y es el método más ampliamente utilizado en los programas de rehabilitación respiratoria. Existe amplia evidencia, en múltiples ensayos clínicos controlados y no controlados realizados en hospitales y en el hogar de los pacientes con EPOC, que los programas de EM-EEII logran aumentar la resistencia a la fatiga y la capacidad de realizar ejercicio ${ }^{13-23}$.

En el meta-análisis, realizado por Lacasse y $\operatorname{cols}^{24}$, evaluaron el efecto de la rehabilitación respiratoria en la capacidad de ejercicio y la calidad de vida de los pacientes con EPOC. Entre los criterios de inclusión, consideraron los estudios en que el entrenamiento físico duraba por lo menos cuatro semanas, con o sin educación, apoyo psicológico, o ambos. Se incluyeron en el análisis 
14 estudios clínicos aleatorizados y controlados. Los autores encontraron una mejoría en la calidad de vida, la magnitud de la disnea, la capacidad de ejercicio funcional (prueba de caminata de 6 minutos) y de la capacidad de esfuerzo máximo (test incremental en cicloergómetro). Estos beneficios del EM-EEII fueron clínicamente importantes.

A partir de este meta-análisis ${ }^{24} \mathrm{y}$ de la reunión de expertos de la Sociedad de Tórax Americana (ATS), en la que se analizó el impacto de la rehabilitación respiratoria (RR) bajo la perspectiva de la medicina basada en la evidencia, quedó establecido que el entrenamiento muscular es el componente de los programas de RR que mayor impacto tiene sobre la disnea y la calidad de vida relacionada con la salud (CVRS) de los pacientes con EPOC, y más concretamente los programas de ejercicio centrados en las extremidades inferiores $^{3}$. Las mejorías clínicas (disminución de la disnea, mejoría de la capacidad de realizar ejercicio y de la calidad de vida) obtenidas en los programas de RR, no se asocian a cambios significativos en la función pulmonar ${ }^{1,2,6}$.

La importancia de la rehabilitación respiratoria no es tan sólo clínica, sino que del punto de vista fisiopatológico Maltais y $\operatorname{cols}^{25}$, documentaron que las muestras de biopsia de músculos de las extremidades inferiores de los pacientes con entrenamiento muscular presentaban una aumento significativo de las enzimas responsables de la función oxidativa muscular (citrato sintetasa y 3-hydroxiacil-CoA deshidrogenasa) y también se reducía la acidosis láctica comparado con el grupo control.

\section{Entrenamiento aeróbico de las extremidades inferiores}

El entrenamiento aeróbico muscular de las extremidades inferiores utilizando grandes masas musculares es el principal factor determinante para el éxito de los programas de rehabilitación respiratoria. Estudios recientes han demostrado que el entrenamiento de las extremidades inferiores de los pacientes con EPOC tiene beneficios clínicos $^{1,6,26,27}$ y también conduce a cambios estructurales y funcionales de la musculatura ${ }^{25}$. Las modalidades de ejercicio más usadas en este tipo de entrenamiento son el cicloergómetro, la cinta rodante, la caminata supervisada con parámetros controlados (velocidad) o una combinación de $\operatorname{estas}^{1,6,28}$.

1. Frecuencia y Duración: La óptima frecuencia $\mathrm{y}$ duración de las sesiones de entrenamiento muscular no ha sido suficientemente precisada, sesiones entre 20 y 40 minutos de ejercicio continuo o a intervalos, entre dos y cinco veces por semana, han sido utilizadas en la mayoría de los programas de rehabilitación. Se ha demostrado que 20 sesiones son suficientes para lograr efectos beneficiosos en los pacientes. Programas más prolongados pueden lograr efectos más duraderos, logrando el máximo beneficio en la sesión número $24^{1,6,29,30}$.

2. Intensidad: El ejercicio de alta intensidad (60 a $80 \%$ del ejercicio máximo alcanzado en el test de cargas incrementales limitado por síntomas) produce importantes ganancias fisiológicas, mejorando la capacidad aeróbica de los pacientes. Los cambios fisiológicos más característicos que producen mejoría en la capacidad aeróbica después de un entrenamiento físico incluyen el aumentos en el número de capilares de la fibra muscular, en la densidad mitocondrial y capacidad oxidativa de las fibras musculares, retardando el comienzo del metabolismo anaeróbico durante el ejercicio. Estos factores llevan a una disminución de la ventilación minuto, frecuencia cardíaca, consumo de oxígeno y producción de lactato para un mismo nivel de ejercicio ${ }^{1,6-8,24,31}$.

Tabla 1. Recomendaciones para realizar entrenamiento muscular de grandes y pequeños grupos musculares

\begin{tabular}{|c|c|c|}
\hline Recomendación & Grandes grupos musculares & Pequeños grupos musculares \\
\hline a) Modalidad & $\begin{array}{l}\text { Cicloergómetro, cinta rodante, caminata supervisada o una } \\
\text { combinación de éstos }\end{array}$ & $\begin{array}{l}\text { Silla de cuádriceps, bandas elásticas, } \\
\text { pesas, entre otras }\end{array}$ \\
\hline b) Intensidad & $\begin{array}{l}\text { Se recomienda el entrenamiento a intervalos, combinando } \\
\text { altas y bajas intensidades. Alta: } 60-80 \% \text { y baja: } 30-40 \% \\
\text { del valor máximo alcanzado en el test de de evaluación }\end{array}$ & $\begin{array}{l}\text { Se recomienda } 50-85 \% \text { de una repe- } \\
\text { tición máxima }\end{array}$ \\
\hline c) Frecuencia & $2-5$ veces por semana & Diariamente \\
\hline d) Duración & $\begin{array}{l}20-24 \text { sesiones en total } \\
\text { Sesiones: entre } 20 \text { y } 40 \text { minutos por sesión }\end{array}$ & \\
\hline
\end{tabular}


Sin embargo, se ha demostrado que los programas de rehabilitación que utilizan ejercicios de baja intensidad también producen mejorías en la tolerancia al ejercicio aún cuando esto acontezca en ausencia o con cambios fisiológicos limitados. Los mecanismos por los cuales la tolerancia al ejercicio mejora después de un entrenamiento de baja intensidad (30-40\% del valor máximo) no están claramente dilucidados. Los factores que pueden jugar un papel en la mejoría de la capacidad de ejercicio son el aumento de la fuerza de la musculatura periférica y respiratoria, mayor eficiencia mecánica para realizar ejercicio debido a una mejor coordinación y acoplamiento neuromuscular, reducción en la hiperinflación dinámica, disminución de la ansiedad y el temor a la disnea y aumento en la motivación ${ }^{32}$.

La óptima intensidad de ejercicio depende de las necesidades individuales de cada paciente y debe representar la mínima intensidad necesaria para producir respuestas clínicamente significativas. Una manera de desarrollar altas intensidades de ejercicio, en pacientes severamente deteriorados, son los programas de entrenamiento a intervalos (alternando ejercicios de alta y baja intensidad). Este tipo de entrenamiento refleja mejor los requerimientos fisiológicos de las actividades de la vida diaria. Se ha demostrado que los beneficios obtenidos por este tipo de entrenamiento son similares a los del ejercicio continuo. No obstante, el entrenamiento a intervalos tiene como ventaja que los pacientes lo realizan con menor sensación de disnea y fatiga muscular de las extremidades. El entrenamiento a intervalos es mejor tolerado y puede ser considerado una alternativa al entrenamiento continuo en estos pacientes ${ }^{1,33,34}$.

\section{Entrenamiento de fortalecimiento muscular (fuerza y resistencia)}

Debido a que la debilidad de los músculos periféricos contribuye a la limitación en el ejercicio de los pacientes respiratorios crónicos, el ejercicio contra resistencia es una forma de entrenamiento en la que intervienen pequeños grupos musculares y ha sido utilizado en muchos estudios de ejercicio en pacientes con EPOC. El entrenamiento de pequeños grupos musculares disminuye la carga ventilatoria durante el ejercicio físico, por lo que puede ser realizado con altas cargas de trabajo, lo que permite aumentar la masa muscular y mejorar la fuerza y resistencia. Para lograr este objetivo se pueden utilizar implementos tales como la silla de cuádriceps, bandas elásticas o pesas. Los programas de EMEEII incluyen dos o tres series de ocho a diez repeticiones cada una, con una carga equivalente entre $50 \%$ y $85 \%$ de una repetición máxima. Por su buena tolerancia este tipo de entrenamiento está particularmente indicado en sujetos que tienen importante atrofia muscular. La combinación de programas de ejercicios de resistencia aeróbica $\mathrm{y}$ fortalecimiento muscular puede aumentar los efectos beneficiosos del ejercicio en los pacientes con $\mathrm{EPOC}^{1,6,35-37}$. En la Tabla 1 se entregan algunas recomendaciones para realizar entrenamiento de EEII en el paciente con EPOC.

\section{Estrategias que permiten incrementar la intensidad del ejercicio}

1. Reducción del trabajo ventilatorio. Intervenciones tales como una óptima terapia broncodilatadora que permita mayores niveles de ventilación o una disminución del requerimiento ventilatorio en un determinado nivel de ejercicio pueden permitir un incremento de la intensidad del entrenamiento. Esto puede mejorar los resultados del mismo especialmente en pacientes con $\mathrm{VEF}_{1} \leq 50 \%$ (etapas III y IV de GOLD) $)^{1,2,24}$.

2. Uso de oxígeno suplementario. El uso de oxígeno suplementario reduce el requerimiento ventilatorio para un mismo nivel de trabajo, aumentando la capacidad de ejercicio máximo, aún en sujetos sin hipoxemia; ya que aumenta la tolerancia al ejercicio disminuyendo la disnea junto con la demanda ventilatoria, reduciendo además el grado de limitación del flujo espiratorio y por consiguiente la hiperinflación $^{38-41}$.

Los programas de entrenamiento muscular en niveles de ejercicio submáximo disminuyen la producción de lactato circulante, lo que se correlaciona con una reducción de la ventilación minuto durante la actividad física. Los pacientes que más se benefician con el uso de oxígeno son aquellos que presentan hipoxemia durante el ejercicio, una menor demanda ventilatoria para una misma carga de trabajo produce mejorías en la disnea y la capacidad de realizar ejercicio.

En resumen, son múltiples los beneficios del uso de oxígeno suplementario durante el ejercicio: a) Evita la hipoxemia durante el ejercicio; b) Disminuye la presión de la arteria pulmonar; c) Mejora la función del ventrículo derecho; d) Disminuye la disnea; e) Disminuye los niveles 
de lactato sérico durante el ejercicio; f) Disminuye la hiperinflación dinámica; g) Disminuye el trabajo diafragmático y retarda su fatiga, y $h$ ) Aumenta la resistencia a la fatiga y la capacidad de realizar ejercicio ${ }^{1,2,24,38,42,43}$.

\section{Grado de recomendación}

El entrenamiento muscular de extremidades inferiores otorga significativos beneficios a los pacientes con EPOC en cuanto a disminuir la disnea, mejorar la capacidad de ejercicio y la calidad de vida (calidad de la evidencia A, recomendación fuerte).

\section{Conclusiones}

Existe suficiente evidencia clínica del beneficio del entrenamiento muscular de extremidades inferiores en los pacientes con EPOC. El entrenamiento muscular aeróbico de EEII está indicado como parte de todo programa de rehabilitación respiratoria dirigido a pacientes con EPOC. La óptima intensidad de ejercicio depende de las necesidades individuales de cada paciente. El entrenamiento a intervalo es mejor tolerado que el ejercicio continuo en este tipo de pacientes. La óptima terapia broncodilatadora y el uso de oxígeno suplementario en los programas de entrenamiento muscular permite a los pacientes con EPOC obtener mayores beneficios en términos de actividad física.

\section{Bibliografía}

1.- NICI L, DONNER C, WOUTERS E, ZUWALLACK R, AMBROSINO N, BOURBEAU J, et al. American Thoracic Society/European Respiratory Society statement on pulmonary rehabilitation. Am J Respir Crit Care Med 2006; 173: 1390-413.

2- KILLIAN K J. Limitation to muscular activity in chronic obstructive pulmonary disease. Eur Respir J 2004; 24: 6-7.

3.- GÜELL R. EPOC y músculos periféricos. Arch Bronconeumol 2000; 36: 519-24.

4.- TROOSTERS T, CASABURI R, GOSSELINK R, DECRAMER M. Pulmonary rehabilitation in chronic obstructive pulmonary disease. Am J Respir Crit Care Med 2005; 172: 19-38.

5.- ROCHESTER C L. Exercise training in chronic obstructive pulmonary disease. J Rehabil Res Dev 2003; 40 (5 Suppl 2): 59-80.

6.- ACCP/AACVPR Pulmonary Rehabilitation Guidelines
Panel. Pulmonary rehabilitation. Joint ACCP/AACVPR Evidence-Based Guidelines. Chest 2007; 131: 4S-51S.

7.- CASABURI R, PORSZASZ J, BURNS M R, CARITHERS E R, CHANG R S, COOPER C B. Physiologic benefits of exercise training in rehabilitation of patients with severe chronic obstructive pulmonary disease. Am J Respir Crit Care Med 1997; 155: 1541-51.

8.- EPOC. Diagnóstico y tratamiento integral. Con énfasis en la rehabilitación pulmonar. Horacio Giraldo editor. Tercera edición, Editorial Médica Panamericana, 2008.

9.- KILLIAN K J, SUMMERS E, JONES N L, CAMPBELL E J. Dyspnea and leg effort during incremental cycle ergometry. Am Rev Respir Dis 1992; 145: 1339-45.

10.- GÜELL R, CASAN P, GIMÉNEZ M. Fatiga muscular periférica y respuesta ventilatoria al esfuerzo en la limitación crónica al flujo aéreo (LCFA). Arch Bronconeumol 1996; 32: 79-84.

11.- GAYAN-RAMÍREZ G, KOULOURIS N, ROCA J, DECRAMER M. Respiratory and skeletal muscles in chronic obstructive pulmonary disease. Eur Respir Mon 2006; 38: 201-23.

12.- BERNARD S, LEBLANC P, WHITTOM F, CARRIER G, JOBIN J, BELLEAU R, et al. Peripheral muscle weakness in patients with chronic obstructive pulmonary disease. Am J Respir Crit Care Med 1998; 158: 629-34.

13.- MAN W D, POLKEY M I, DONALDSON N, GRAY B J, MOXHAM J. Community pulmonary rehabilitation after hospitalisation for acute exacerbations of chronic obstructive pulmonary disease: randomised controlled study. BMJ 2004; 329: 1209.

14.- CASABURI R. Exercise training in chronic obstructive lung disease. In: Principles and Practice of Pulmonary Rehabilitation. Casaburi R, Petty TL, editors. Saunders, Philadelphia, 1993.

15.- SINCLAIR D J, INGRAM C G. Controlled trial of supervised exercise training in chronic bronchitis. $\mathrm{Br}$ Med J 1980; 280: 519-21.

16.- COCKCROFT A E, SAUNDERS M J, Berry G. Randomised controlled trial of rehabilitation in chronic respiratory disability. Thorax 1981; 36: 200-3.

17.- O'DONNELL D E, WEBB K A, MCGUIRE M A. Older patients with COPD: benefits of exercise training. Geriatrics 1993; 48: 59-62.

18.- TOSHIMA M T, KAPLAN R M, RIES A L. Experimental evaluation of rehabilitation in chronic obstructive pulmonary disease: short-term effects on exercise endurance and health status. Health Psychol 1990; 9: 237-52.

19.- O'HARA W J, LASACHUK B P, MATHESON P, RENAHAM M C, SCHOLTTER D G, LIKER E S. Weight training and backpacking in chronic obstructive pulmonary disease. Respir Care 1984; 29: 1202-10.

20.- WIJKSTRA P J, VAN ALTENA R, KRAAN J, OTTEN V, POSTMA D S, KOËTER G H. Quality of life in patients with chronic obstructive pulmonary disease improves after rehabilitation at home. Eur Respir J 1994; 7: 269-73. 
21.- GOLDSTEIN R S, GORT E H, STUBBING D, AVENDANO M A, GUYATT G H. Randomised controlled trial of respiratory rehabilitation. Lancet 1994; 344: 1394-7.

22.- GÜELL R, CASAN P, BELDA J, SANGENIS M, MORANTE F, GUYATT G H, et al. Long-term effects of outpatient rehabilitation of COPD: A randomized trial. Chest 2000; 117: 976-83.

23.- O'SHEA S D, TAYLOR N F, PARATZ J D. Progressive resistance exercise improves muscle strength and may improve elements of performance of daily activities for people with COPD: a systematic review. Chest 2009; 136: 1269-83.

24.- LACASSE Y, WONG E, GUYATT G H, KING D, COOK D J, GOLDSTEIN R S. Meta-analysis of respiratory rehabilitation in chronic obstructive pulmonary disease. Lancet 1996; 348: 1115-9.

25.- MALTAIS F, LEBLANC P, SIMARD C, JOBIN J, BÉRUBÉ C, BRUNEAU J, et al. Skeletal muscle adaptation to endurance training in patients with chronic obstructive pulmonary disease. Am J Respir Crit Care Med 1996; 154: 442-7.

26.- BRITISH THORACIC SOCIETY Standards of Care Subcommittee on Pulmonary Rehabilitation. Pulmonary rehabilitation. Thorax 2001; 56: 827-34.

27.- LACASSE Y, BROSSEAU L, MILNE S, MARTIN $\mathrm{S}$, WONG E, GUYATT G H, et al. Pulmonary rehabilitation for chronic obstructive pulmonary disease. Cochrane Database Syst Rev 2002; (3): CD003793.

28.- COOPER C B. Exercise in chronic pulmonary disease: aerobic exercise prescription. Medicine \& Science in Sports \& Exercise 2001; 33: 671-9.

29.- ÁLVAREZ HERNÁNDEZ J. Enfoque terapéutico global de la disfunción muscular en la EPOC. Nutr Hosp 2006; 21 (Supl 3): 76-83.

30.- SOLANES I, GÜELL R, CASAN P, SOTOMAYOR C, GONZÁLEZ A, FEIXAS T, et al. Duration of pulmonary rehabilitation to achieve a plateau in quality of life and walk test in COPD. Respir Med 2009; 103: 722-8.

31.- CASABURI R, ZUWALLACK R. Pulmonary rehabilitation for management of chronic obstructive pulmonary disease. N Engl J Med 2009; 360: 1329-35.

32.- DATTA D, ZUWALLACK R. High versus low intensity exercise training in pulmonary rehabilitation: is more better? Chron Respir Dis 2004; 1: 143-9.
33.- BEAUCHAMP MK, NONOYAMA M, GOLDSTEIN RS, HILL K, DOLMAGE TE, MATHUR S, BROOKS D. Interval versus continuous training in individuals with chronic obstructive pulmonary disease--a systematic review. Thorax 2010; 65: 157-64.

34.- NASIS I G, VOGIATZIS I, STRATAKOS G, ATHANASOPOULOS D, KOUTSOUKOU A, DASKALAKIS A, et al. Effects of interval-load versus constantload training on the BODE index in COPD patients. Respir Med 2009; 103: 1392-8.

35.- SANTIWORAKUL A, JARUNGJITAREE S, JALAYONDEJA W, CHANTAROTHORN S, SUPAIBULPIPAT S. Effect of lower extremity exercise on muscle strength and physical capacity in COPD patients. J Med Assoc Thai 2009; 92: 556-63.

36.- STORER T W. Exercise in chronic pulmonary disease: resistance exercise prescription. Med Sci Sports Exerc 2001; 33 (7 Suppl): S680-92.

37.- NICI L, ZUWALLACK R. Pulmonary rehabilitation: today and tomorrow. Breathe 2010; 6: 305-11.

38.- SOMFAY A, PORSZASZ J, LEE S M, CASABURI R. Dose-response effect of oxygen on hyperinflation and exercise endurance capacity in nonhypoxaemic COPD patients. Eur Respir J 2001; 18: 77-84.

39.- O'DONNELL D E, D'ARSIGNY C, WEBB K A. Effects of hyperoxia on ventilatory limitation during exercise in advanced chronic obstructive pulmonary disease. Am J Respir Crit Care Med 2001; 163: 892-8.

40.- DEAN N C, BROWN J K, HIMELMAN R B, DOHERTY J J, GOLD W M, STULBARG M S. Oxygen may improve dyspnea and endurance in patients with chronic obstructive pulmonary disease and only mild hypoxemia. Am Rev Respir Dis 1992; 146: 941-5.

41.- WOODCOCK A A, GROSS E R, GEDDES D M. Oxygen relieves breathlessness in "pink puffers". Lancet 1981; 1: 907-9.

42.- EMTNER M, PORSZASZ J, BURNS M, SOMFAY A, CASABURI R. Benefits of supplemental oxygen in exercise training in nonhypoxemic chronic obstructive pulmonary disease patients. Am J Respir Crit Care Med 2003; 168: 1034-42.

43.- NICI L, RASKIN J, ROCHESTER C L, BOURBEAU J C, CARLIN B W, CASABURI R, et al. Pulmonary rehabilitation: What we know and what we need to know. J Cardiopulm Rehabil Prev 2009; 29: 141-51.
Correspondencia a:

Diego Vargas B.

Unidad de Kinesiología, Instituto Nacional del Tórax.

Av. J. M. Infante 717, Providencia.

Santiago, Chile.

E-mail: dvargasbruce@gmail.com 